$\begin{array}{lll}\text { Chao Ping Wu, MD } & \text { Mani Latifi, MD } & \text { Eduardo Mireles-Cabodevila, MD } \\ \text { Department of Critical Care, } & \text { Department of Critical Care, } & \text { Department of Critical Care, Respiratory Institute, } \\ \text { Respiratory Institute, } & \text { Respiratory Institute, } & \begin{array}{l}\text { Cleveland Clinic; Assistant Professor, } \\ \text { Cleveland Clinic }\end{array} \\ \text { Cleveland Clinic } & \begin{array}{l}\text { Cleveland Clinic Lerner College of Medicine } \\ \text { of Case Western Reserve University, Cleveland, } \mathrm{OH}\end{array}\end{array}$

\title{
Management of patients with COVID-19 in the MICU
}

\section{ABSTRACT}

COVID-19 management practices devised for the medical intensive care unit are centered on 2 main goals: ensuring caregiver safety and providing the highest quality patient care through adherence to evidence-based best practices. Rapid, sweeping changes for successful management are based on creating an educational platform to introduce and then further cement these concepts through a unified approach to clinical care. Creating a culture change in a short period of time requires overcoming a host of challenges; however, the result is a more unified and focused approach.

\section{KEY POINTS}

Use personal protective equipment based on the risk of transmission when in contact with patients who are potentially COVID-19-positive.

Evaluate patients often to avoid delaying intubation.

If intubation is required, modify procedures to ensure caregiver safety.

On admission, obtain electrocardiography, troponin levels, ferritin levels, and select serologic tests.

Bundle all care (eg, medications, laboratory samples, and procedures) to limit traffic into the room.

To minimize information overload, create a team to review available literature and develop an easily accessible and up-to-date educational resource.

Dr. Mireles-Cabodevila has disclosed intellectual property rights (royalties or patent sales) with Jones \& Bartlett Learning and Super Duper Publications.

doi:10.3949/ccjm.87a.ccc017
A S THE COVID-I9 pandemic continues, it is A essential for healthcare providers to follow updated literature and adapt these to individual institutions. In this review, we describe the COVID-19 management practices devised for the medical intensive care unit (MICU) in the Respiratory Institute at Cleveland Clinic.

The foundation of our MICU operations is centered on 2 main goals: ensuring caregiver safety, and providing the highest quality patient care through adherence to evidencebased best practices.

\section{ENSURING CAREGIVER SAFETY}

We need to preserve our workforce for the health of the community and the functioning of the institution. Identifying the appropriate situations for personal protection equipment (PPE) is essential, so we adapted evolving standards of care (Figure 1) that outline when and how PPE should be used based on the risk of transmission when in contact with potential COVID-19-positive patients, either confirmed or under investigation for infection. Equipment includes a surgical mask, gown, protective eyewear, and gloves for all caregiver interactions. An N95 respirator or a powered air-purifying respirator (PAPR) is used for encounters with patients undergoing therapies at high risk for aerosolization (eg, high-flow nasal cannula [HFNC], noninvasive ventilation [NIV]) or procedures at high risk for disease transmission (eg, intubation, tracheostomy, endoscopy).

In addition, to reduce the high risk of transmission during the process of donning or doffing PPE, especially when doffing, we instituted a "buddy" system to create an additional layer of caregiver safety (Figure 2). This per- 


\section{Personal Protective Equipment (PPE) Recommendations for Patient Care (Inpatient \& Ambulatory)}

\begin{tabular}{|c|c|c|c|c|c|}
\hline $\begin{array}{l}\text { Gloves } \\
\text { Gowns }\end{array}$ & $\dot{\bullet}$ & $\begin{array}{l}\text { Face Mask } \\
\text { Eye Protect }\end{array}$ & & & \\
\hline \multicolumn{2}{|c|}{$\begin{array}{l}\text { Guidelines for Care of SUSPECTED OR CONFIRMED COVID-19 } \\
\text { patients: }\end{array}$} & & & & \\
\hline $\begin{array}{l}\text { - Confirmed positive COVID-19 } \\
\text { - Signs or symptoms of respirato- } \\
\text { ry illness } \\
\text { - Pending respiratory viral testing } \\
\text { - Presumed positive }\end{array}$ & $\begin{array}{l}\text { Inpatient setting: Patients are } \\
\text { immediately placed in appropriate } \\
\text { isolation precautions } \\
\text { (Droplet/Contact + Eyewear) }\end{array}$ & $\begin{array}{l}\text { N95 } \\
\text { (fit-tested } \\
\text { respirator) }\end{array}$ & $\begin{array}{l}\text { Face Mask² } \\
\text { (surgical or } \\
\text { ear-loop mask) }\end{array}$ & \begin{tabular}{|} 
Protective \\
Eyewear \\
$\begin{array}{c}\text { (goggles or face } \\
\text { shield) }\end{array}$
\end{tabular} & $\begin{array}{l}\text { Gown \& } \\
\text { Gloves }\end{array}$ \\
\hline \multicolumn{2}{|c|}{ Ambulatory Setting: Caregivers Actively Seeing Patients } & & $v$ & & Gloves Only \\
\hline \multicolumn{2}{|c|}{$\begin{array}{l}\text { Inpatient Setting: Caregivers Actively Seeing Patients } \\
\text { PPE for Oxygen Therapy Transport Guide for Clinicians }\end{array}$} & & $\sqrt{ }$ & & \\
\hline \multicolumn{2}{|c|}{$\begin{array}{l}\text { Collection of Nasopharyngeal/Oropharyngeal Swab for Respiratory Viral } \\
\text { Specimen Test (including COVID-19) }\end{array}$} & & $\sqrt{ }$ & & \\
\hline \multicolumn{2}{|c|}{$\begin{array}{ll}\text { Aerosol Generating Procedures (AGPs): } \\
\begin{array}{ll}\text { - Bronchoscopy } & \text { - ENT or Gl endoscopies } \\
\text { - Nebulization } & \text { - TEE } \\
\text { - NIPPV } & \text { - High flow } 02 \\
\text { - Open tracheal suctioning } & \text { - Naso-enteric tube placement } \\
\text { - Intubation/extubation } & \end{array}\end{array}$} & & & & \\
\hline \multirow{2}{*}{\multicolumn{2}{|c|}{ 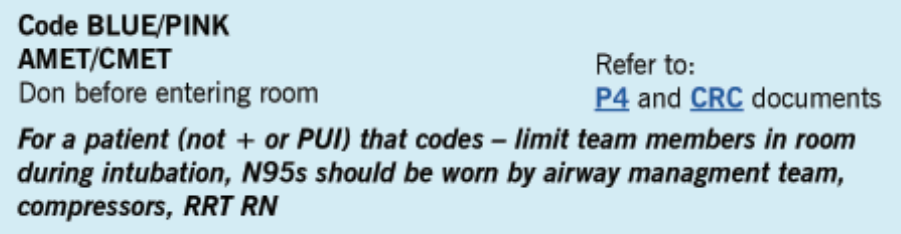 }} & & & & \\
\hline & & & & & \\
\hline $\begin{array}{l}\text { Powered Air Purifying Respirator } \\
\text { (PAPR) indicated for caregivers for whom } \\
\text { an N95 mask does not fit. }\end{array}$ & $\begin{array}{l}\text { ace mask usage: } \\
\text { Masks should always be worn covering nose } \& \\
\text { Handle used mask by ear loops or strings ONL } \\
\text { Never touch front of the mask }\end{array}$ & \multicolumn{4}{|c|}{$\begin{array}{l}\text { - Perform hand hygiene after handling used mask } \\
\text { - Fabric and cloth masks ARE NOT considered PPE }\end{array}$} \\
\hline
\end{tabular}

Figure 1. Cleveland Clinic recommendation for personal protective equipment for COVID-19 (updated July $28,2020)$.

son provides direct observation and feedback during the process of donning and doffing PPE to ensure caregiver safety.

We also adjusted respiratory practices to enhance safety by minimizing aerosolization. For hospitalized patients with COVID-19 who develop hypoxemia, oxygen is supplemented with a target oxygen saturation $\left(\mathrm{SpO}_{2}\right)$ range of $90 \%$ to $96 \%$, and the patients are transferred to the MICU on escalating requirements for closer observation, as they can quickly dete- riorate. In patients with increasing oxygen requirements, we prefer HFNC with a surgical mask placed on the patient, as tolerated, to minimize aerosolization. In patients with concomitant comorbid conditions that indicate the use of NIV with either continuous positive airway pressure (CPAP) or bilevel positive airway pressure (BiPAP), such as chronic obstructive pulmonary disease or congestive heart failure, we use expiratory-port high-energy particulate air (HEPA) filters. 


\section{COVID-19: BUDDY SYSTEM PROTOCOLS}

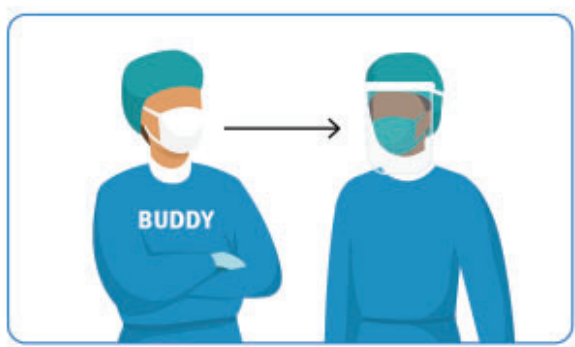

Ask your buddy to watch you don PPE before you enter the patient's room to make sure you are in compliance.

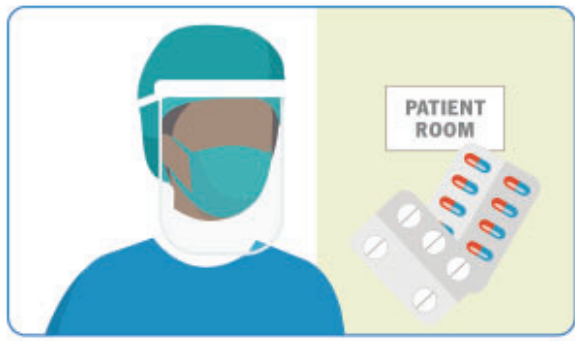

When your patient needs medications, ask an available buddy to add the medications to the IV poles outside of the room, while you assist the patient inside.

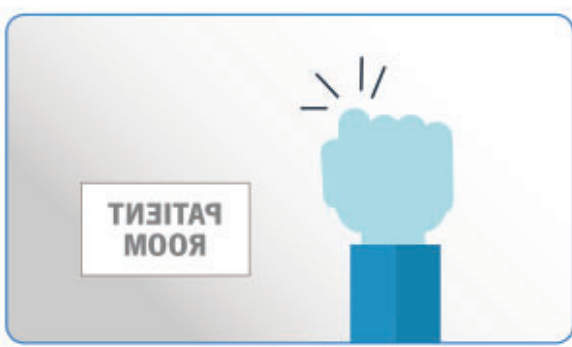

Need supplies? Knock on the glass while you are in the room to alert an available buddy. Open the door for them, and have them hand you the supplies.

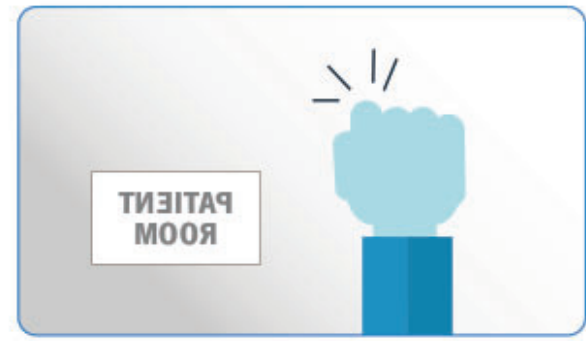

When you're ready to exit, knock on the window to alert an available buddy. Doff to minimize risk of error. early intuba-

tion, these

patients are

evaluated often

to avoid

delaying

intubation
Please note: The buddy system was designed to keep patients and caregivers safe. For a full list of PPE recommendations for a clinical setting, visit clevelandclinic.org/COVID19.

Figure 2. Cleveland Clinic buddy system protocol.

We integrate objective criteria into clinical judgment to identify patients with potential deterioration while on NIV or HFNC (Table 1). Although we do not advocate early intubation, these patients are evaluated often to avoid delaying intubation. In patients who are intubated, we use inline nebulizers. In those not on mechanical ventilation, we use metered-dose inhalers.

Procedures for intubation, if required, have also been modified to ensure caregiver safety. Preoxygenation is achieved with delivery of $100 \%$ oxygen via a nonrebreather mask or HFNC. Bag mask ventilation is not recommended. We have our most experienced operators use video laryngoscopy to perform intubation to minimize the duration of the procedure and to ensure maximum distance from the patient's oropharynx. After successful intubation, we put patients directly on mechanical ventilators with continuous capnometer monitoring. 
Chest radiography is performed at admission to ensure placement for necessary procedures such as nasogastric tube and central line. However, we avoid daily chest radiography unless clinically indicated, such as when changing location of the endotracheal tube or changing ventilation parameters (eg, increasing resistance, hypoxemia). We developed a process for performing portable radiologic studies from outside the room.

\section{MAINTAIN BEST PATIENT CARE}

It is challenging to maintain our standards of care when isolation practices increase. We have 5 distinct MICUs and we dedicated 2 of these units (with plans to expand further based on patient volume) to the COVID-19 patient population. Each patient has his or her own room with distinct walls.

To ensure the safety of other patients and our caregivers, we created a cohort unit in which we place all confirmed COVID-19 patients, while all MICU admissions were tested for COVID-19 regardless of the diagnosis.

Given that there is no curative therapy for COVID-19 and that therapeutic considerations have been extrapolated from limited experience and evolving literature, we developed multidisciplinary teams to help develop consistent clinical practice strategies. These teams include MICU providers, infectious disease specialists, and pharmacists.

For hypoxic respiratory failure, our mechanical ventilation strategy includes low-tidal-volume ventilation with a goal end-inspiratory plateau pressure of $30 \mathrm{~cm}$ $\mathrm{H}_{2} \mathrm{O}$ or below and allows for permissive hypercarbia $(\mathrm{pH} \geq 7.15)$. We titrate the fraction of inspired oxygen and positive endexpiratory pressure $\left(\mathrm{FiO}_{2} / \mathrm{PEEP}\right)$ according to established protocols. ${ }^{1}$ It is often easier to use existing evidence-based protocols in a pandemic when unfamiliar teams may be caring for these patients. Early proning and neuromuscular blockade are recommended as adjuvant therapy in patients. For patients who are refractory to conventional mechanical ventilation, trials of salvage therapy with inhaled vasodilator and extracorporeal life support can be considered. After initial
TABLE 1

\section{Signs of respiratory failure despite noninvasive ventilation or a high-flow nasal cannula}

\section{Patients on noninvasive ventilation}

Tidal volume $>9.5 \mathrm{~mL} / \mathrm{kg}$ ideal body weight consistently over the first 4 hours

Fraction of inspired oxygen $\left(\mathrm{FiO}_{2}\right)>60 \%$ to maintain target oxygen saturation $\left(\mathrm{SpO}_{2}\right)$

\section{Patients on high-flow nasal cannula}

ROX index (ratio of $\mathrm{SpO}_{2} / \mathrm{FiO}_{2} /$ respiratory rate) $\geq 4.88$ at 2, 6, and 12 hours is a good predictor of no need for intubation, and $<3.85$ predicts high risk of need for intubation

volume resuscitation, as patients often come with evidence of volume depletion, we are vigilant in preventing and decreasing volume overload. In patients with acute lung injury, diuresis with the Fluid and Catheter Treatment Trial lite protocol should be implemented. ${ }^{2}$ Dexamethasone is given to COVID-19 patients requiring mechanical ventilation or oxygen supplementation if there is no contraindication. ${ }^{3}$

Although uncommon, bacterial co-infections have been reported in patients with COVID-19. ${ }^{3}$ As a preventive measure, we start coverage with antibiotics in critically ill patients presenting with severe respiratory distress, basing it on their risk factors for community vs drug-resistant organisms (eg, MRSA, Pseudomonas). Procalcitonin is ordered on admission and followed to help with de-escalation of antibiotic therapy.

Critically ill patients with COVID-19 experience a sequelae of manifestations from activation of the innate inflammatory cascade, which increases the incidence of cardiomyopathy/heart failure, disseminated intravascular coagulation, venous thromboembolism (VTE), and the cytokine-release syndrome. Although there are currently no accepted standardized therapies for the prevention or treatment of these phenomena, we perform screening modalities that include electrocardiography, troponin levels, ferritin, and select serologic tests on admission with follow-up based on the patient's 


\section{TABLE 2}

\section{Cleveland Clinic MICU COVID-19 workup checklist (updated 7/25/2020)}

All patients under investigation or with confirmed COVID-19 on admission: procalcitonin, full respiratory viral panel, 2 sets of blood cultures, human chorionic gonadotropin (females of reproductive age)

\section{Patients with confirmed COVID-19 or strongly suspected}

On admission:

Complete blood cell count (CBC) with differential, complete metabolic panel, liver function tests, lactate dehydrogenase, C-reactive protein (CRP), fibrinogen, D-dimer, high-sensitivity troponin, $\mathrm{N}$-terminal pro-B-type natriuretic peptide, creatine kinase (CK), CK-MB, procalcitonin, ferritin, activated partial thromboplastin time, international normalized ratio, interleukin 6

Chest radiography (bundle with other care)

Electrocardiography

Point-of-care ultrasonography

Echocardiography if shock or suspicion for cardiomyopathy

Deep vein thrombosis scan if $D$-dimer $>3,000 \mathrm{ng} / \mathrm{mL}$

Daily monitoring:

CBC, complete metabolic panel, magnesium, phosphate

CRP daily; if elevated along with hemodynamic instability or persistent fever, consider checking triglyceride, ferritin, fibrinogen, and liver function tests; daily CRP can be discontinued at discretion of providers

Troponin every day for 3 days or if change in hemodynamics

No routine chest radiography

Corrected QT interval from cardiac monitor

clinical course (Table 2). In patients at high risk of VTE (ie, those with D-dimer $>$ 3,000 ng/mL fibrinogen equivalent units), we perform point-of-care ultrasonography to assess the presence of thromboembolism over extremities. Therapeutic anticoagulation is indicated for patients with conclusive evidence of VTE.

As with any new disease that has unproven therapies, our approach is to develop the best evidence-based guidelines for our teams to follow and to engage in clinical trials to form better guidance.

We also have developed bundle care practices to preserve PPEs while maintaining our standards of care. We bundle all care (eg, giving medications, obtaining laboratory samples, and performing procedures at the same time) to limit traffic into the room to only what is essential. Our nursing and respiratory therapy teams have placed medication administration pumps and ventilator screens outside of patient rooms, which has decreased our PPE use by about $50 \%$ to $60 \%$. This placement also allows for more efficient titration of medications and ventilator settings, resulting in decreased sedative use and easier adjustment of ventilator support.

To further limit room traffic, we delegate 1 caregiver to perform a daily comprehensive physical examination with a dedicated singleuse stethoscope that remains in the room. The results are documented and shared with all other care and consulting teams to limit entrance. They are repeated based on changes in the patient's clinical condition.

Neurologic assessment and skin examination are performed by bedside nurses every 2 to 4 hours with other bundled care. All other practices including daily routine lab draws and ancillary support such as physical therapy continue on an essential-only basis.

Family visitation is limited as an infectioncontrol method, but the team has placed special emphasis on maintaining communication with patients and their supporting members. Updates are amended in various fashions based on the provider and patient's preference to be done outside of the rooms. This involves phone calls, videoconferencing, and in some instances communicating by writing on the glass doors.

Be aware of information overload. Not a minute goes by without a new post, tweet, e-mail, or letter from caregivers at the front lines with new disease manifestations or unproven therapies. At times, this generates an overwhelming amount of anxiety. We have created an educational team that is responsible for reviewing all available literature and developing an educational platform that serves our teams. This is done through an easily accessible, shared toolkit that allows our caregivers to rapidly find protocols and up-to-date educational resources (eg, webinars, simulations, checklists), especially with rapidly updated guidelines. This resource becomes the source of truth for the institution, aligns caregivers, and decreases anxiety from misinformation. 


\section{WU AND COLLEAGUES}

\section{SUMMARY}

To manage COVID-19, we have developed best practices for the MICU to maintain the highest quality patient care while ensuring the safety of all caregivers. This requires rapid, sweeping changes to the system. Success is based on creating an educational platform

\section{REFERENCES}

1. Acute Respiratory Distress Syndrome Clinical Network. Mechanical ventilation protocol summary. Accessed August 14, 2020. http:// www.ardsnet.org/files/ventilator_protocol_2008-07.pdf

2. Grissom CK, Hirshberg EL, Dickerson JB, et al; National Heart Lung and Blood Institute Acute Respiratory Distress Syndrome Clinical Trials Network. Fluid management with a simplified conservative protocol for the acute respiratory distress syndrome. Crit Care Med 2015; 43(2):288-295. doi:10.1097/CCM.0000000000000715 to introduce and then further cement these concepts through a unified approach to clinical care. Creating a culture change in a short period of time requires overcoming a host of challenges. However, the result is a more unified and focused approach.

3. The RECOVERY Collaborative Group; Horby P, Lim WS, Emberson JR, et al. Dexamethasone in hospitalized patients with Covid-19 preliminary report. N Engl J Med 2020 Jul 17; doi:10.1056/NEJMoa2021436

4. Arentz M, Yim E, Klaff L, et al. Characteristics and outcomes of 21 critically ill patients with COVID-19 in Washington State. JAMA 2020; 323(16):1612-1614. doi:10.1001/jama.2020.4326

Address: Eduardo Mireles-Cabodevila, MD, Pulmonary Medicine, A90, Cleveland Clinic, 9500 Euclid Avenue, Cleveland, $\mathrm{OH} 44195$; MIRELEE@ccf.org 